\title{
Implication of Neurological Pupil Index for Monitoring of Brain Edema
}

\author{
Tae Jung Kim ${ }^{1,2}$ and Sang-Bae Ko ${ }^{1,2}$ \\ ${ }^{1}$ Department of Neurology, ${ }^{2}$ Department of Critical Care Medicine, Seoul National University Hospital, Seoul, Korea
}

Dear Editor:

Malignant middle cerebral artery (MCA) infarction is associated with poor neurologic outcome due to its high risk for brain swelling and transtentorial herniation $[1,2]$. Standard treatment for intracranial pressure (ICP) crisis in malignant MCA infarction includes osmotic therapy and decompressive hemicraniectomy. When surgical decompression is not indicated, targeted temperature management (TTM) can be used as an alternative option [2]. TTM is an effective therapeutic option for reducing brain edema, which was proven to improve functional outcome in patients with malignant MCA infarction [3,4]. However, TTM often requires light sedation to minimize shivering, which possibly limits early detection of neurologic worsening. For continuous neurological assessment, serial pupillary examination has been performed in the neurological intensive care unit (NICU). However, its poor interrater reliability raises a need for a more reliable and objective tool for pupillary evaluation [5]. Pupillometer exam allows to quantitatively measure pupillary reactivity. In addition, it provides with more information on exact pupil size, latency, constriction velocity, and Neurological Pupil Index (NPi) value, which enable us to continuously assess different aspects of pupillary light reflex [6-8]. Here, we report a case with a malignant MCA infarction treated on TTM. While on TTM, continuous assessment on neurologic status was possible using an automated pupillometer.

A 78-year-old man was presented with left side weakness and drowsy mentality on awakening. This patient was found normal 6 hours before the presentation. $\mathrm{He}$ was a smoker, and had a history of uncontrolled hypertension. On presentation, this patient was found to have atrial fibrillation. Neurologic examination showed severe weakness on the left (Medical Research Council grade 1) with gaze preponderance to the right. National Institutes of Health Stroke Scale (NIHSS) score was 12 and
Received on March 30, 2017

Revised on June 2, 2017

Accepted on June 4, 2017

Sang-Bae Ko

Department of Neurology, Seoul National University Hospital, 101 Daehak-ro, Jongno-gu, Seoul 03080, Korea Tel: $+82-2-2072-2278$ Fax: $+82-2-3672-7553$ E-mail: sangbai1378@gmail.com

*No potential conflict of interest relevant to this article was reported. 
Glasgow Coma Scale (GCS) was 14 (E3V5M6). Brain magnetic resonance imaging revealed an infarction in the right MCA territory with right proximal internal carotid artery occlusion. Thrombolytic therapy was not considered due to unclear onset of symptom with extensive infarction without diffusion-perfusion mismatch. The patient was transferred to the Stroke Unit for close monitoring. On hospital day 2 , his neurological status was aggravated to NIHSS of 16 and GCS of 12 (E3V3M6). Follow-up brain computed tomography (CT) showed that the midline was shifted $5 \mathrm{~mm}$ to the left side. After thorough discussion with family members, they rejected decompressive hemicraniectomy. The patient was transferred to the NICU for intensive neuromonitoring.

Serial pupillary reactivity was assessed using NPi-100 pupillometer (NeurOptics, Irvine, CA, USA). Initial pupillometer findings showed that latency was prolonged (right/left, 0.25/0.43 seconds) and NPi value was decreased (right/left, 4.1/3.0) on the left compared to the right. However, other parameters were within normal
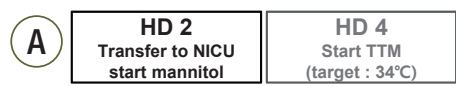

\begin{tabular}{|c|c|}
\hline HD 10 \\
$\begin{array}{c}\text { Start rewarming } \\
\text { (TTM day 6) }\end{array}$ & $\begin{array}{c}\text { HD 11 } \\
\text { Aggravated symptom } \\
\text { Resume TTM }\end{array}$ \\
\hline
\end{tabular}

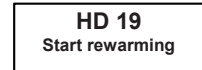

start mannitol $\left(\right.$ target : $\left.34^{\circ} \mathrm{C}\right)$

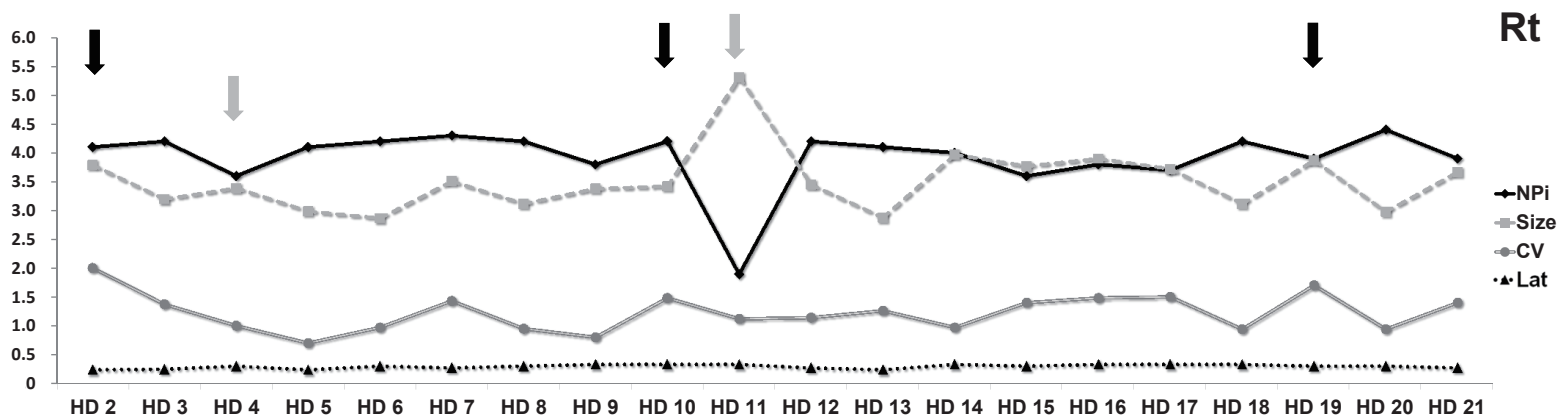

HD 2 HD 3 HD 4 HD 5 HD 6 HD 7 HD 8 HD 9 HD 10 HD 11 HD 12 HD 13 HD 14 HD 15 HD 16 HD 17 HD 18 HD 19 HD 20 HD 21

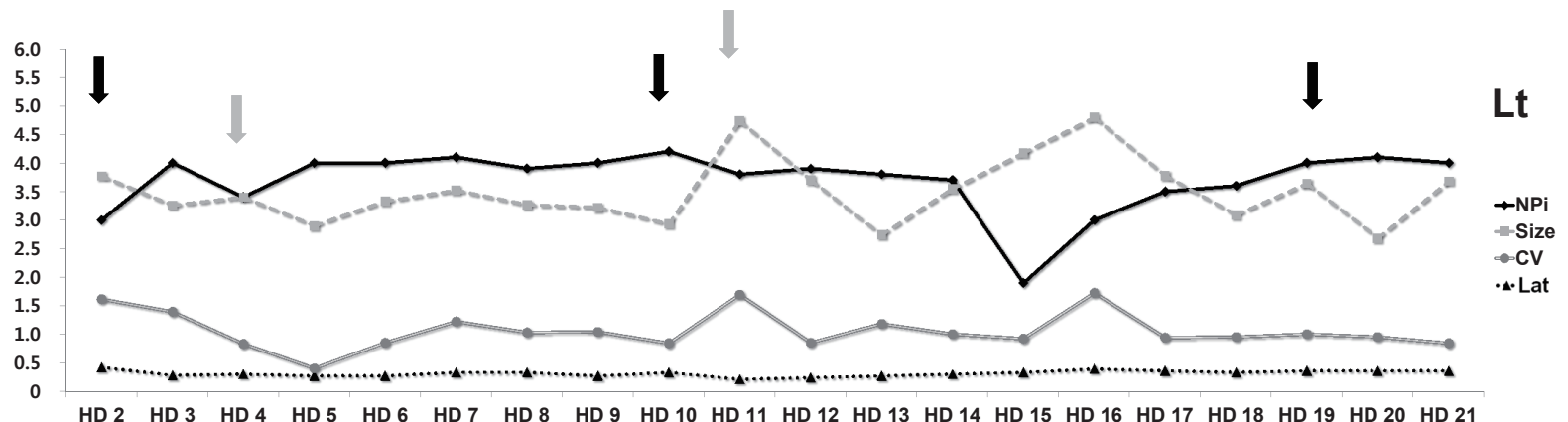

(B)

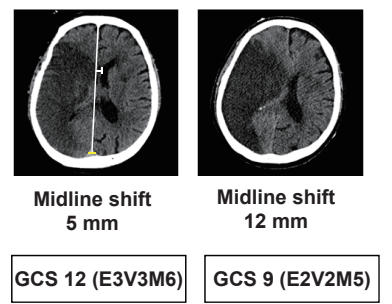

(C)

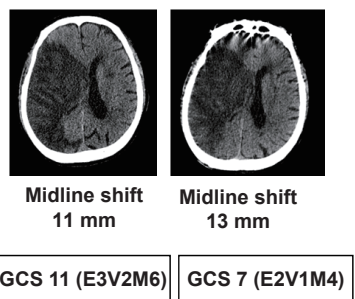

(D)

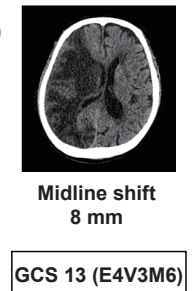

Figure 1. Serial pupillometer parameters and brain CT of the patient. (A) Serial pupillometer parameters of the patient. (B-D) Serial brain CTs of the patient. Serial changes of pupillometer parameters: on hospital day (HD) 4, bilateral NPi value decreased and neurological symptom was aggravated combining increased midline shifting $(A)$. Cerebral edema was gradually decreased during TTM and pupillometer parameters were stably maintained until HD 10. On HD 11, bilateral NPIs suddenly decreased below 2 and brain CT scan showed that brain edema was aggravated (midline shift $13 \mathrm{~mm}$ ) (C). TTM was restarted and maintained until HD 19 with continuous pupillometer monitoring. HD: hospital day; NICU: neurological intensive care unit; TTM: targeted temperature management; Rt: right; NPi: Neurological Pupil Index; CV: constriction velocity; Lat: latency; Lt: left; GCS: Glasgow Coma Scale; CT: computed tomography. 
ranges (pupil size, $3.79 / 3.77 \mathrm{~mm}$; constriction velocity, $2.01 / 1.62 \mathrm{~mm} / \mathrm{sec}$, right/left, respectively), despite with radiological signs of midline shift (reference values in normal: latency $<0.24$ to 0.28 seconds; constriction velocity $>0.8 \mathrm{~mm} / \mathrm{sec}$; NPi value, 3 to 5 ) (Figure 1) $[9,10]$. Osmotic therapy ( $20 \%$ mannitol, $0.5 \mathrm{~g} / \mathrm{kg}$ every 8 hours) was initiated and left NPi value was improved to 4.0 (Figure 1). While on osmotic therapy, pupillometer parameters were well maintained above 3.9. On day 4 , however, bilateral NPi values suddenly decreased to 3.6/3.4 (right/left) and NIHSS increased to 24 and GCS was aggravated to 9 (E2V2M5). Follow-up brain CT showed that midline shift was aggravated to $12 \mathrm{~mm}$.

TTM at $34^{\circ} \mathrm{C}$ was initiated to mitigate brain swelling and herniation with permission. TTM was achieved by a surface cooling device (Arctic Sun; Medivance, Louisville, CO, USA). Anti-shivering protocol was also maintained in accordance with Columbia protocol including skin counter warming, high-dose buspirone, magnesium sulfate infusion, intermittent bolus injection of pethidine, and continuous infusion of remifentanil (at a rate of 0.02 to $0.06 \mu \mathrm{g} / \mathrm{kg} / \mathrm{min}$ ) [11]. After initiating TTM, the NPi values were improved (right/left, 4.2/4.0). On hospital day 10, TTM day 6, brain CT scan was confirmed that midline shift was slightly improved and controlled rewarming was initiated at a rate of $0.05^{\circ} \mathrm{C} / \mathrm{h}$, targeting to $36^{\circ} \mathrm{C}$ (Figure 1). On hospital day 11, bilateral NPi values suddenly dropped to 1.9 (right) and 1.2 (left) and GCS decreased to 7 (E2V1M4), which raised a concern for rebound brain edema. Follow-up brain CT scan showed that midline was shift to $13 \mathrm{~mm}$, suggesting aggravation of brain edema (Figure 1). Rewarming was stopped and TTM was resumed targeting at $35^{\circ} \mathrm{C}$. While on the second stage of TTM, bilateral pupillometer parameters remained stable. After confirming neurological stability, controlled rewarming was resumed on hospital day 19. The patient survived and was transferred to the Department of Rehabilitation Medicine with a NIHSS total score of 16 and a modified Rankin scale score of 5 .

This case illustrated that neurological status could be successfully monitored using an automated pupilometer in a patient with malignant MCA infarction on TTM. Oculomotor nerve is prone to stretching and compression in transtentorial herniation, and abnormal pupillary reflex is associated with brain swelling and poor neurologic outcome [6,7]. Therefore, bedside examination of light reflex is a key neurologic examination in the NICU. However, assessment of pupillary reflex using a penlight has multiple limitations in terms of its low concordance rate among the examiners and low reproducibility [5]. By contrast, automated pupillometer exam offers more reliable, safe, and repeatable results for pupillary reactivity. In addition to the pupil reactivity, automated pupillometer provides more information on pupil parameters such as the maximal/minimal pupil size, percentage change in constriction, latency, constriction velocity, dilatation velocity, and NPi value $[6,7,12]$. Among them, NPI value has been frequently used in the practice for estimating ICP crisis $[6-8,12]$. NPi value was automatically standardized in a scale of 0 to 5 , greater value suggesting more prompt light reflex. In general, NPi value $<3$ indicates an abnormal pupillary light reflex, which is linked to a critical ICP elevation and herniation $[6-8,12]$. Therefore, monitoring of NPi value may be useful for monitoring impending transtentorial herniation in patients with ICP crisis.

In this case, we performed pupillometer examination in every hour, and a sudden drop of NPi or pupillary constriction velocity prompted us to initiate treatment for brain swelling and herniation. Interestingly, NPi score was lower on the left side even though this patient has MCA infarction on the right. This could be due to Kernohan's notch phenomenon, which is common in patients with massive brainstem compression due to brain edema [13]. We think that pupillometer examination was helpful in limiting permanent brain damage in patients with transtentorial herniation. Sedative agents or opioid drugs are known to affect the pupillary response [14]. However, administered doses of remifentanil or pethidine were not significantly different when pupillometer parameters were changed. Moreover, bolus infusion of pethidine as a treatment for shivering did not lead to sudden change 
in pupillary reflexes. Therefore, we do not think that the changes of NPI were simply due to sedative drugs.

Here, we report a case of malignant cerebral infarction treated on TTM. An accurate and objective measurement of pupillary reflex using automated pupilometer was helpful in continuous assessing neurological status, which decreased the time delay to initiate appropriate intervention for brain swelling. NPi values obtained using automated pupillometer may serve as a useful tool for accurate measurement of pupillary reactivity in the NICU.

\section{ORCID}

Tae Jung Kim http://orcid.org/0000-0003-3616-5627

Sang-Bae Ko

\section{REFERENCES}

1. Hacke W, Schwab S, Horn M, Spranger M, De Georgia $\mathrm{M}$, von Kummer R. 'Malignant' middle cerebral artery territory infarction: clinical course and prognostic signs. Arch Neurol 1996;53:309-15.

2. Wijdicks EF, Sheth KN, Carter BS, Greer DM, Kasner SE, Kimberly WT, et al. Recommendations for the management of cerebral and cerebellar infarction with swelling: a statement for healthcare professionals from the American Heart Association/American Stroke Association. Stroke 2014;45:1222-38.

3. Lakhan SE, Pamplona F. Application of mild therapeutic hypothermia on stroke: a systematic review and meta-analysis. Stroke Res Treat 2012;2012:295906.

4. Su Y, Fan L, Zhang Y, Zhang Y, Ye H, Gao D, et al. Improved neurological outcome with mild hypothermia in surviving patients with massive cerebral hemispheric infarction. Stroke 2016;47:457-63.

5. Olson DM, Stutzman S, Saju C, Wilson M, Zhao W,

Aiyagari V. Interrater reliability of pupillary assessments. Neurocrit Care 2016;24:251-7.

6. Fountas KN, Kapsalaki EZ, Machinis TG, Boev AN, Robinson JS, Troup EC. Clinical implications of quantitative infrared pupillometry in neurosurgical patients. Neurocrit Care 2006;5:55-60.

7. Chen JW, Gombart ZJ, Rogers S, Gardiner SK, Cecil S, Bullock RM. Pupillary reactivity as an early indicator of increased intracranial pressure: the introduction of the Neurological Pupil index. Surg Neurol Int 2011;2:82.

8. Couret D, Boumaza D, Grisotto C, Triglia T, Pellegrini L, Ocquidant $\mathrm{P}$, et al. Reliability of standard pupillometry practice in neurocritical care: an observational, double-blinded study. Crit Care 2016;20:99.

9. Martínez-Ricarte F, Castro A, Poca MA, Sahuquillo J, Expósito L, Arribas M, et al. Infrared pupillometry: basic principles and their application in the non-invasive monitoring of neurocritical patients. Neurologia 2013;28:41-51.

10. Ellis CJ. The pupillary light reflex in normal subjects. Br J Ophthalmol 1981;65:754-9.

11. Choi HA, Ko SB, Presciutti M, Fernandez L, Carpenter AM, Lesch C, et al. Prevention of shivering during therapeutic temperature modulation: the Columbia anti-shivering protocol. Neurocrit Care 2011;14:389-94.

12. Zafar SF, Suarez JI. Automated pupillometer for monitoring the critically ill patient: a critical appraisal. J Crit Care 2014;29:599-603.

13. Kernohan JW, Woltman HW. Incisura of the crus due to contralateral brain tumor. Arch Neurol Psychiatry 1929;21:274-87.

14. Shirozu K, Setoguchi H, Tokuda K, Karashima Y, Ikeda M, Kubo M, et al. The effects of anesthetic agents on pupillary function during general anesthesia using the automated infrared quantitative pupillometer. $\mathrm{J}$ Clin Monit Comput 2017;31:291-6. 